International Journal of Pure and Applied Mathematics

Volume 108 No. 4 2016, 809-818

ISSN: 1311-8080 (printed version); ISSN: 1314-3395 (on-line version)

url: http://www.ijpam.eu

doi: 10.12732 /ijpam.v108i4.7

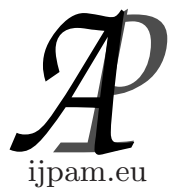

\title{
PARTITION DIMENSION OF HONEYCOMB DERIVED NETWORKS
}

\author{
Chris Monica M. ${ }^{1}$, S. Santhakumar ${ }^{2}$ \\ ${ }^{1,2}$ Department of Mathematics \\ Loyola College \\ Chennai, 600 034, INDIA
}

\begin{abstract}
For a vertex $v$ of a connected graph $G$ and a subset $S$ of $V(G)$, the distance between $v$ and $S$, denoted by $d(v, S)$, is $\min \{d(v, x) \mid x \in S\}$. Let $\Pi=\left\{S_{1}, S_{2} \ldots S_{\mathrm{k}}\right\}$ be an ordered $k$-partition of $V(G)$. The representation of $v$ with respect to $\Pi$ is the $k$-vector $r(v \mid \Pi)=$ $\left(d\left(v, S_{1}\right), d\left(v, S_{2}\right) \ldots d\left(v, S_{\mathrm{k}}\right)\right)$. The $k$-partition is a resolving partition if the $k$-vectors $r(v \mid \Pi)$, for all $v \in V(G)$ are distinct. The minimum $k$ for which there is a resolving $k$-partition of $V(G)$ is called the partition dimension $p d(G)$ of $G$. In this paper, we determine partition dimension of Hive network, Honeycomb rhombic mesh, Honeycomb rectangular mesh.
\end{abstract}

AMS Subject Classification: $05 \mathrm{C} 12$

Key Words: resolving partition, partition dimension, hive network, honeycomb rhombic mesh, honeycomb rectangular mesh

\section{Introduction}

The vertices of a connected graph are represented by partitions of vertex set into many subsets where the distances between each vertex and subsets in the partition are calculated. Based on this concept, resolving partition of a graph has been introduced [2]. This concept has wide applications in chemistry, problems of pattern recognition, image processing and navigation of robots in networks $[3,13]$.

For $v \in V(G)$ and $S \subset V(G)$, the distance $d(v, S)$ between $v$ and $S$ is defined as $d(v, S)=\min \{d(v, x) \mid x \in S\}$. The representation of $v$ with respect to $\Pi$ is a $k$-vector $r(v \mid \Pi)=\left(d\left(v, S_{1}\right), d\left(v, S_{2}\right) \ldots d\left(v, S_{k}\right)\right)$, where $\Pi$ is an ordered

Received: January 19, 2016

Published: August 16, 2016

$\S$ Correspondence author (c) 2016 Academic Publications, Ltd.

url: www.acadpubl.eu 
$k$-partition $\left\{S_{1}, S_{2}, \ldots S_{k}\right\}$ of $V(G)$ and $v \in V(G)$. The partition $\Pi$ is called a resolving partition for $G$ if distinct vertices of $G$ have distinct representation with respect to $\Pi$. The minimum $k$ for which there is a resolving $k$-partition of $V(G)$ is a partition dimension $p d(G)$ of $G$ and a resolving partition of $V(G)$ containing $p d(G)$ elements is called a minimum resolving partition $[1,2,6,15]$.

For a nontrivial connected graph $G, p d(G) \leq \operatorname{dim}(G)+1[2,4]$ and in case the order of $G$ is $n, n \geq 2$, then $p d(G)$ is 2 if and only if $G=P_{n}$ [2]. The partition dimension $p d(G)$ of a graph $G$ with order $n$ is $n$ if and only if $G=K_{n}$. Chartrand et al [2] proved that for a graph $G$ which is neither a path nor a complete graph with order $n \geq 4,3 \leq p d(G) \leq n-1$.

The partition dimension of an $n$-cycle, Petersen graph, 3 -cube are 3,4 and 3 respectively $[2,15]$. Partition dimension problem has been studied for circulant networks, hexagonal and honeycomb networks [1], tree [12], cartesian product [10], gear, helm, sunflower and friendship graph [8].

\section{Honeycomb Network}

Honeycomb network $H C(n)$ is obtained from $H C(n-1)$ by adding a layer of hexagons around the boundary of $H C(n-1)$.

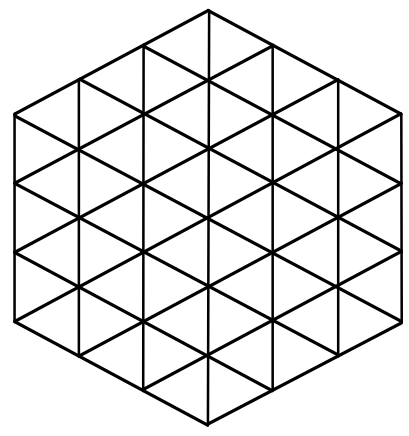

(a)

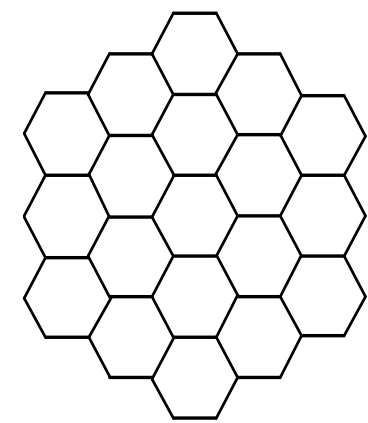

(b)

Figure 1: (a) 4-dimensional Hexagonal Network, (b) 3-dimensional Honeycomb Network

The parameter $n$ of $H C(n)$ is determined by the number of hexagons between the centre and boundary of $H C(n)$. The number of vertices and edges of $H C(n)$ are $6 n^{2}$ and $9 n^{2}-3 n$ respectively. The diameter is $4 n-1$. A 3-dimensional Honeycomb Network is depicted in Figure 1(b). 
Honeycomb networks are widely used in computer graphics, cellular phone base stations, image processing and for representation of benzenoid hydrocarbons in chemistry [11].

An Hexagonal network $H X(n)$ of dimension $n$ has $3 n^{2}-3 n+1$ vertices and $9 n^{2}-15 n+6$ edges, where $n$ is the number of vertices on one side of the hexagon. A 4-dimensional Hexagonal network is depicted in Figure 1(a). The bounded dual of $H X(n)$ is $H C(n-1)$ [5].

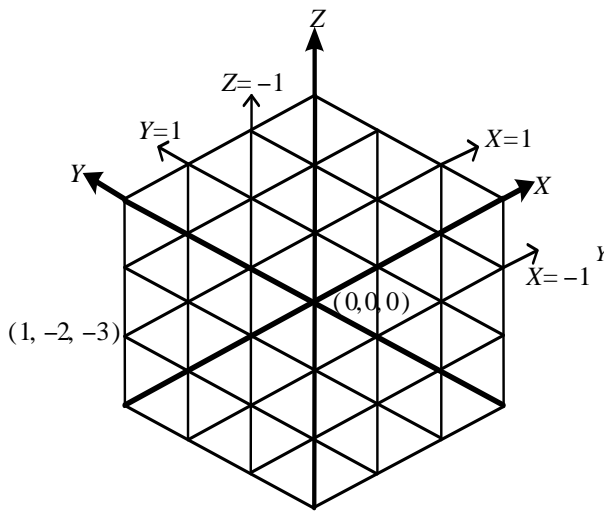

(a)

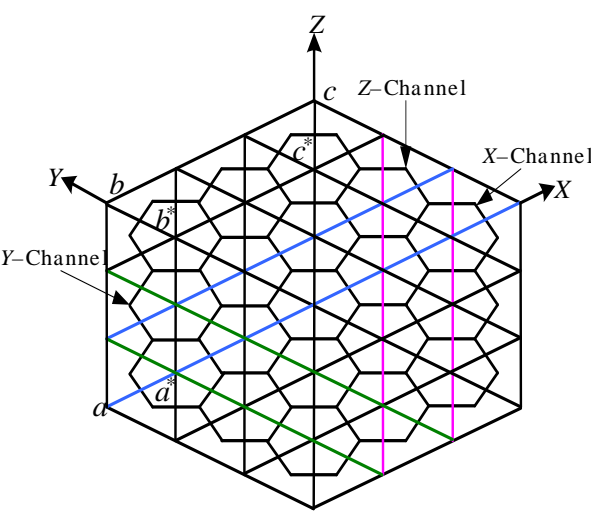

(b)

Figure 2: (a) Coordinate system in $H X(4)$, (b) Channels in $H X(4)$

Stojmenovic [11] proposed a coordinate system for a honeycomb network. This was adapted by Paul et al [14] for assigning coordinates to the vertices in the hexagonal network. In this scheme, three axes $X, Y$ and $Z$ parallel to three edge directions and at mutual angle of 120 degrees between any two of them are introduced as in the Figure 2(a). Lines parallel to the coordinate axes are called as $X$-lines, $Y$-lines and $Z$-lines. Here $X=h$ and $X=-k$ are two $X$-lines on either side of the $X$-axis. Any vertex of $H X(n)$ is assigned with coordinates $(x, y, z)$ in the above scheme. A segment of an $X$-line in the Hexagonal network consisting of vertices $(x, y, z)$, with $x$ coordinate fixed is denoted by $P_{X}$. ie., $P_{X}=\left\{\left(x_{0}, y, z\right) / y_{1} \leq y \leq y_{2}, z_{1} \leq z \leq z_{2}\right\}$. Similarly, $P_{Y}=\left\{\left(x, y_{0}, z\right) / x_{1} \leq\right.$ $\left.x \leq x_{2}, z_{1} \leq z \leq z_{2}\right\}$ and $P_{Z}=\left\{\left(x, y, z_{0}\right) / x_{1} \leq x \leq x_{2}, y_{1} \leq y \leq y_{2}\right\}$ are the $Y$-line and $Z$-line of a hexagonal network respectively [14]. See Figure 2(a).

An $X$-channel, denoted by $C_{X}$ is the strip between any consecutive $X$ lines in $H X(n)$. Similarly, $C_{Y}$ and $C_{Z}$ are defined. It can be easily seen that, for two vertices $\left(x_{1}, y_{1}, z_{1}\right)$ and $\left(x_{2}, y_{2}, z_{2}\right)$ of $H C(n), x_{1}=x_{2}$ in an $X$-channel. This is applicable for $Y$-channel and $Z$-channel too. The $X$-channel between $P_{X, n}$ and $P_{X, n-1}$ is represented by $C_{X, n}[1] . N_{r}(v)$ is the set of vertices at a distance $r$ from the vertex $v$ of $H C(n)$. See Figure $2(\mathrm{~b})$. 
Lemma 1. [1] $N_{r}\left(a^{*}\right) \subset C_{Y}$ o $C_{Z}$, where $a^{*}$ is the vertex in honeycomb.

Lemma 2. [1] For any $r_{1}$ and $r_{2}, N_{r_{1}}\left(a^{*}\right) \cap N_{r_{2}}\left(C_{X, n}\right)$ is either empty or singleton.

Theorem 3. [1] Let $G$ be a honeycomb network $H C(n)$. Then $p d(G)=3$.

\section{Hive Network}

Increase in computational power demands a new design for parallel computing and the most preferable networks are the ones with less complexity than others. Hive network has less complexity.

A $t$-dimensional hive network $H N(t)$ is composed of $2 t-1$ interconnected honeycomb networks of dimension $n$ and adjacent honeycombs are connected with additional vertical edges.

A vertex in the hive network is addressed by four integer coordinates $(x, y, z$, $v)$. The $v$ coordinate indicates the position of a honeycomb in the hive network. The value $v$ is zero for the $t^{t h}$ honeycomb in hive network.

The value of $v$ will be subsequent positive integers for the honeycombs which are located in the upward direction from the $t^{\text {th }}$ honeycomb. Similarly, the value of $v$ will be subsequent negative integers for the honeycombs which are located in the downward direction from the $t^{t h}$ honeycomb. A vertex in hive network has only one vertical edge but two extreme layers in hive network does not have a vertical edge for every second vertex [9].

Lemma 4. Let $G$ be a hive network $H N(t)$. Then $p d(G)>3$.

Proof. Hive network of size $t$ is composed of $2 t-1$ interconnected honeycomb network and $p d(H C(n))=3[1]$. Thus $p d(G)>3$. 


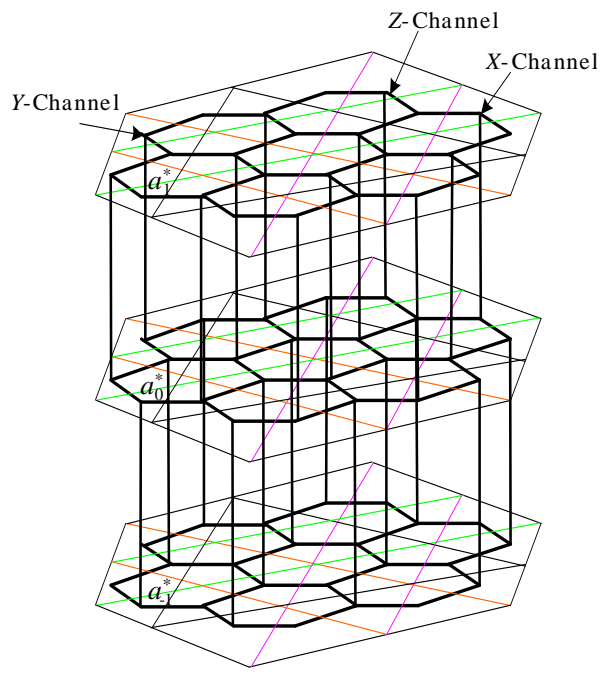

Figure 3: Channels in $H N(2)$.

$H C_{i}(n)$ denotes the $i^{t h}$ honeycomb of dimension $n$ in the hive network $H N(t)$ and $a_{i}^{*},(t-1 \leq i \leq 1-t)$ denotes a vertex in $H C_{i}(n) . C_{X_{\mathrm{i}}}, C_{Y_{\mathrm{i}}}$ and $C_{Z_{\mathrm{i}}}$-denotes the $X$ channel, $Y$ channel and $Z$ channel in the $i^{\text {th }}$ honeycomb of hive network respectively. A hive network $H N(2)$ with channels are depicted in Figure 3.

Lemma 5. For any $r_{1}$ and $r_{2}, N_{r_{1}}\left(a_{i}^{*}\right) \cap N_{r_{2}}\left(C_{X_{\mathbf{i}}, n}\right)$ is either empty or singleton.

Theorem 6. Let $G$ be a hive network $H N(t), t \geq 2$. Then $p d(G)=4$.

Proof. Let $S_{1}=\left\{a_{1-t}^{*}\right\}, S_{2}=\left\{a_{i}^{*}, t-1 \leq i \leq 2-t\right\}, S_{3}=\left\{C_{X_{\mathrm{i}}, n}, t-1 \leq i \leq\right.$ $1-t\}, S_{4}=V(G)-\left\{S_{1} \cup S_{2} \cup S_{3}\right\}$. Let $u=\left(x_{1}, y_{1}, z_{1}, v_{1}\right)$ and $v=\left(x_{2}, y_{2}, z_{2}, v_{2}\right)$ be two vertices of $G$.

Claim: $\Pi \doteq\left\{S_{1}, S_{2}, S_{3}, S_{4}\right\}$ is a resolving 4-partition of $G$.

We consider two cases, namely when $u$ and $v$ are in the same honeycomb and $u$ and $v$ are in different honeycomb in hive network.

Case $\left\{v_{1}=v_{2}\right\}$

Subcase $\left\{x_{1}=x_{2}\right\}$ : Then $u, v \in C_{X_{\mathrm{i}}}$ and $d\left(u, a_{1-t}^{*}\right) \neq d\left(v, a_{1-t}^{*}\right)$. Thus $d\left(u, S_{1}\right) \neq d\left(v, S_{1}\right)$.

Subcase $\left\{y_{1}=y_{2}\right\}$ : In this case, $u, v \in C_{Y_{\mathrm{i}}}$. For $u, v \in N_{r_{1}}\left(a_{i}^{*}\right), d\left(u, S_{3}\right) \neq$ $d\left(v, S_{3}\right)$. Suppose $d\left(u, S_{3}\right)=d\left(v, S_{3}\right)$, then $u, v \notin N_{r_{1}}\left(a_{i}^{*}\right)$ which would imply that $d\left(u, S_{2}\right) \neq d\left(v, S_{2}\right)$ whenever $t-1 \leq i \leq 2-t$ and $d\left(u, S_{1}\right) \neq d\left(v, S_{1}\right)$ when $i=1-t$. 
Subcase $\left\{z_{1}=z_{2}\right\}$ : Here, $u, v \in C_{Z_{\mathrm{i}}}$. Let $u, v \in N_{r_{1}}\left(a_{i}^{*}\right)$. If $d\left(u, S_{1}\right)$ $=d\left(v, S_{1}\right)$ then $d\left(u, S_{3}\right) \neq d\left(v, S_{3}\right)$. Suppose $d\left(u, S_{3}\right)=d\left(v, S_{3}\right)$ then $u, v \notin$ $N_{r_{1}}\left(a_{i}^{*}\right)$ which is a contradiction.

Subcase $\left\{x_{1} \neq x_{2}, y_{1} \neq y_{2}, z_{1} \neq z_{2}\right\}$ : Suppose that $d\left(u, S_{2}\right)=d\left(v, S_{2}\right)$ for $t-1 \leq i \leq 2-t$ or $d\left(u, S_{1}\right)=d\left(v, S_{1}\right)$ for $i=1-t$, then $u, v \in N_{r_{1}}\left(a_{i}^{*}\right)$ for some $r_{1}$. Thus $d\left(u, S_{3}\right) \neq d\left(v, S_{3}\right)$.

Case $\left\{v_{1} \neq v_{2}\right\}$ : Then $u \in H C_{i}(n)$ and $v \in H C_{j}(n), i \neq j$, which results in the following subcases.

Subcase $\left\{x_{1}=x_{2}\right\}$ : Either $d\left(u, S_{1}\right)=d\left(v, S_{1}\right)$ or $d\left(u, S_{1}\right) \neq d\left(v, S_{1}\right)$. Suppose $d\left(u, S_{1}\right)=d\left(v, S_{1}\right)$, then $u \in N_{r_{1}}\left(a_{i}^{*}\right) \subset H C_{i}(n)$ and $v \in N_{r_{2}}\left(a_{j}^{*}\right) \subset$ $H C_{j}(n)$, where $r_{1} \neq r_{2}$. Thus $d\left(u, S_{2}\right) \neq d\left(v, S_{2}\right)$.

Subcase $\left\{y_{1}=y_{2}\right\}$ : Which leads to the fact that either $d\left(u, S_{1}\right) \neq d\left(v, S_{1}\right)$ or $d\left(u, S_{2}\right) \neq d\left(v, S_{2}\right)$.

Similarly, we can prove for $z_{1}=z_{2}$.

Subcase $\left\{x_{1} \neq x_{2}, y_{1} \neq y_{2}, z_{1} \neq z_{2}\right\}$ : In this case, either $d\left(u, S_{1}\right) \neq d\left(v, S_{1}\right)$ or $d\left(u, S_{3}\right) \neq d\left(v, S_{3}\right)$.

Hence $p d(G)=4$.

\section{Honeycomb Rhombic Mesh}

A Honeycomb Rhombic Mesh of size $n$, denoted by $\operatorname{HRoM}(n)$, has $n$ vertices on each line to the boundary of rhombus. The number of edges of $\operatorname{HRoM}(n)$ is $3 n^{2}-2 n$. The diameter of $\operatorname{HRoM}(n)$ is $4 n-3[11]$. Figure 4 shows $\operatorname{HRoM}(6)$.

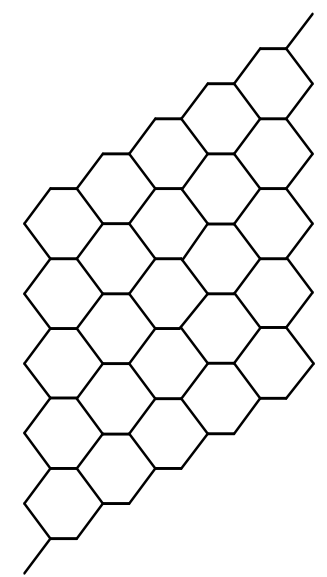

Figure 4: $\operatorname{HRoM(6).~}$ 
We partition the vertices of $H \operatorname{RoM}(n)$ into $n$ segments, namely $B_{1}, B_{2} \ldots B_{n}$ where each $B_{i}$ consists of $2 n$ vertices as shown in Figure 5.

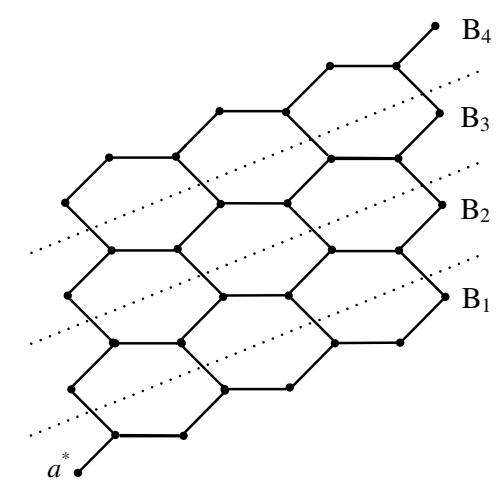

Figure 5:HRoM(4) with 4 segments

Lemma 7. Let $N_{r}\left(a^{*}\right)=\left\{u_{i}\right\}, 1 \leq i \leq k$, for some $k$. If $u_{p}, u_{q} \in N_{r}\left(a^{*}\right)$, then $u_{p}$ and $u_{q}$ will not be in the same segment $B_{i}$.

Proof. It is true that $d\left(u, a^{*}\right) \neq d\left(v, a^{*}\right)$ for all $u, v \in B_{i}$. Thus any two vertices in $B_{i}$ are not equidistant from $a^{*}$.

The following lemma states the distance between a vertex and a segment.

Lemma 8. If $v \in B_{i}$, then $d\left(v, B_{j}\right)=|i-j|$.

According to lemma 7 , no two vertices in the same segments are equidistant from $a^{*}$ which helps to formulate the following lemma.

Lemma 9. For any $r_{1}$ and $r_{2}, N_{r_{1}}\left(a^{*}\right) \cap N_{r_{2}}\left(B_{i}\right)$ is either empty or singleton.

Theorem 10. Let $G=H \operatorname{RoM}(n)$. Then $p d(G)=3$.

Proof. Let $S_{1}=\left\{a^{*}\right\}, S_{2}=\left\{b_{1}\right\}, S_{3}=V(G)-\left\{S_{1} \cup S_{2}\right\}$. Let $u$ and $v$ be any two vertices of $G$.

Claim: $\Pi=\left\{S_{1}, S_{2}, S_{3}\right\}$ is a resolving 3-partition of $G$.

Case $\left\{u, v \in B_{i}\right\}$ : In view of lemma $8, d\left(u, S_{2}\right)=d\left(v, S_{2}\right)$ but $d\left(u, S_{1}\right) \neq$ $d\left(v, S_{1}\right)$ by lemma 7 .

Case $\left\{u \in B_{i}, v \in B_{j}, i \neq j\right\}$ : Then $d\left(u, S_{2}\right) \neq d\left(v, S_{2}\right)$.

Thus, no two vertices in $G$ will have identical representation with respect to $\Pi$. Hence $\operatorname{pd}(G)=3$. 


\section{Honeycomb Rectangular Mesh}

Honeycomb Rectangular $\operatorname{Mesh}(H R e M)$ has two parameters, the length and breadth of two sides of rectangle which is denoted by $\operatorname{HRe} M(m, n)$, where $m$ and $n$ denotes length and breadth respectively. The number of edges of $\operatorname{HRe} M(m, n)$ is $3 m n-m-n$ and the diameter of $\operatorname{HRe} M(m, n)$ is $2 n+m-2$ for $2 n \geq m$ and $2 m-2$, otherwise [11].

$\operatorname{HRe} M(m, n)$ is partitioned into $2 n$ segments $B_{1}, B_{2} \ldots B_{2 n}$ where each $B_{i}$ consists of $n$ vertices. $\operatorname{HRe} M(4,4)$ with 8 segments is shown in Figure 6.

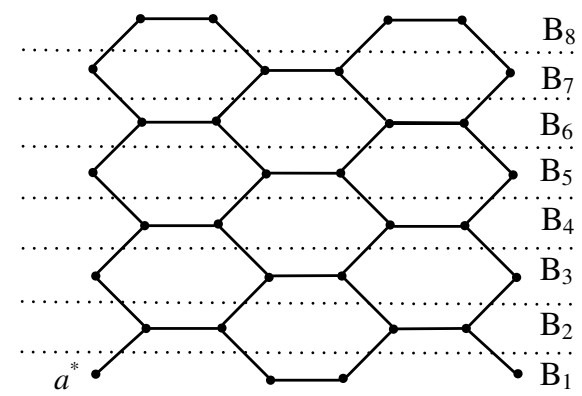

Figure 6: $\operatorname{HRe} M(4,4)$ with 8 segments

The following lemmas are formulated based on the particular pattern of partition shown in Figure 6.

Lemma 11. Let $N_{r}\left(a^{*}\right)=\left\{u_{i}\right\}, 1 \leq i \leq k$, for some $k$. If $u_{p}, u_{q} \in N_{r}\left(a^{*}\right)$, then $u_{p}$ and $u_{q}$ will not be in the same segment $B_{i}$.

Lemma 12. If $v \in B_{i}$, then $d\left(v, B_{j}\right)=|i-j|$.

Lemma 13. For any $r_{1}$ and $r_{2}, N_{r_{1}}\left(a^{*}\right) \cap N_{r_{2}}\left(B_{i}\right)$ is either empty or singleton.

Theorem 14. Let $G=H \operatorname{Re} M(m, n)$. Then $\operatorname{pd}(G)=3$.

Proof. Let $S_{1}=\left\{a^{*}\right\}, S_{2}=\left\{b_{1} \cup b_{2}\right\}, S_{3}=V(G)-\left\{S_{1} \cup S_{2}\right\}$.

Claim: $\Pi=\left\{S_{1}, S_{2}, S_{3}\right\}$ is a resolving 3-partition of $G$.

Case $\left\{u, v \in B_{i}\right\}$ : In this case, $d\left(u, S_{2}\right)=d\left(v, S_{2}\right)$ but $d\left(u, S_{1}\right) \neq d\left(v, S_{1}\right)$ by lemmas 11,12 .

Case $\left\{u \in B_{i}, v \in B_{j}, i \neq j\right\}$ : Then $d\left(u, S_{2}\right) \neq d\left(v, S_{2}\right)$.

Thus, no two vertices in $G$ will have identical representation with respect to $\Pi$. Hence $\operatorname{pd}(G)=3$. 


\section{Conclusion}

Hive network, Honeycomb rhombic mesh, Honeycomb rectangular mesh networks have considerable advantage when compare to other networks in terms of degree, diameter, total number of edges, costs, bisection width, etc. The partition dimension of these networks have been studied in this paper.

\section{Acknowledgements}

This research work is supported by UGC-NFOBC F./2014-15/NFO-2014-15OBC-TAM-6048 of the University Grants Commission, New Delhi, India.

\section{References}

[1] Bharati Rajan, Albert William, Indra Rajasingh, Cyriac Grigorious and Sudeep Stephen, On Certain Networks with Partition Dimension Three, Proceedings of the International Conference on Mathematics in Engineering and Business Management, (2012), 169-172.

[2] Chartrand. G, Salehi. E, Zhang. P, The Partition Dimension of Graphs, Aequations Mathematicae, 59(1-2), (2000), 45-54, doi: 10.1007/PL00000127.

[3] Chartrand. G, Eroh. L, Johnson. M. A, Oellermann. O. R, Resolvability in Graphs and Metric Dimension of a Graph, Discrete Applied Mathematics, 105, (2000), 99-113, doi: 10.1016/S0166-218X(00)00198-0.

[4] Chappell. G, Gimbel. J, Hartman. C, Bounds on the Metric and Partition Dimension of a Graph, Ars Combinatoria, 88, (2008), 349-366.

[5] Fabian Garcý Julio Solano, Ivan Stojmenovic, Milos Stojmenovic, "Higher Dimensional Hexagonal Networks", J. Parallel Distrib. Comput., 63, (2003), 1164-1172, doi: 10.1016/j.jpdc.2003.07.001.

[6] Fehr. M, Gosselin. S, Oellermann. O. R. The Partition Dimension of Cayley Digraphs, Aequations Mathematicae, 71, (2006), 1-18, doi: 10.1007/s00010-005-2800-z.

[7] Harary. F, Melter. R. A, On the Metric Dimension of a Graph, Ars Combinatoria, 2, (1976), 191-195.

[8] Imran Javaid, Sara Shokat, On the Partition Dimension of some Wheel related Graphs, Journal of Prime Research in Mathematics, 4, (2008), 154-164.

[9] Ireneusz Szczśniak, "The Hive Network and Its Routing Algorithm", Archive of Theoretical and Applied Informatics, 16, (2004), 171-179.

[10] Ismael G. Yero, Juan A. Rodriguez-Velázquez, A Note On the Partition Dimension of Cartesian Product Graphs, Applied Mathematics and Computation, 217, (2010), 35713574, doi: 10.1016/j.amc.2010.08.038.

[11] Ivan Stojmenovic, Honeycomb Networks: Topological Properties and Communication Algorithms, IEEE Transactions on Parallel and Distributed Systems, 8, (1997), 10361042, doi: 10.1109/71.629486. 
[12] Juan A. Rodriguez-Velázquez, Ismael G. Yero, Magdalena Lemanska, On the Partition Dimension of Trees, Discrete Applied Mathematics, 166, (2014), 204-209, doi: 10.1016/j.dam.2013.09.026.

[13] Khuller. S, Raghavachari. B, Rosenfeld. A, Landmarks in Graphs, Discrete Applied Mathematics, 70, (1996), 217-229, doi: 10.1016/0166-218X(95)00106-2.

[14] Paul Manuel, Bharati Rajan, I. Rajasingh, Chris Monica. M, On Minimum Metric Dimension of Honeycomb Networks, Journal of Discrete Algorithms, 6, (2008), 20-27, doi: 10.1016/j.jda.2006.09.002.

[15] Saenpholphat. V, Zhang. P, Conditional Resolvability in Graphs-a Survey, International Journal of Mathematics and Mathematical Sciences, 38, (2004), 1997-2017, doi: 10.1155/S0161171204311403. 